\title{
Análisis Sensorial como una Herramienta Clave para Innovar en la Industria Vinícola
}

\section{Sensory Analysis as a Key Tool to Innovate in Wine Industry}

\author{
María Alicia Peña González 1 https://orcid.org/0000-0002-7734-3816 \\ Silvia Peña González ${ }^{2}$ https://orcid.org/0000-0002-9830-4496 \\ Jorge García Zumalacarregui ${ }^{2}$ https://orcid.org/0000-0002-0130-1230 \\ ${ }^{1}$ Universidad del Azuay, Cuenca, Ecuador \\ mpenag@uazuay.edu.ec
}

${ }^{2}$ Universidad de Cuenca, Cuenca, Ecuador

Silvia.penageucuenca.edu.ec

jorge.garciaz@ucuenca.edu.ec

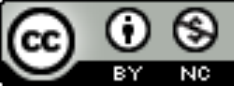

Enviado:

$2021 / 01 / 14$

Aceptado:

$2021 / 02 / 12$

Esta obra está bajo una licencia internacional

Publicado:

$2021 / 06 / 30$

Creative Commons Atribución-NoComercial 4.0.

\section{Resumen}

La innovación y el desarrollo de productos dentro de la industria alimentaria resultan clave para lograr la supervivencia de las empresas, no obstante, estos procesos requieren ser sometidos a actividades de validación que permitan disminuir el riesgo de fracaso de nuevos alimentos en el mercado. En este contexto, el análisis sensorial resulta una herramienta importante para medir el grado de aceptabilidad en los consumidores de un producto alimenticio. La presente investigación evalúa las características sensoriales de tres tipos de productos vinícolas, sometidos a un proceso de microfiltración frente a sus similares pasteurizados, con el objetivo de establecer criterios de control de calidad y grado de aceptación del mercado. Para este desarrollo, se validaron los jueces sensoriales de la empresa vinícola y, posteriormente, se aplicaron pruebas discriminativas y descriptivas sensoriales a las muestras en estudio. Adicionalmente, se realizaron controles microbiológicos y de turbidez. A partir de los resultados obtenidos, se puede observar que hubo diferencias para los atributos: intensidad olfativa e intensidad gustativa en el vino tinto; no obstante, en los demás parámetros sensoriales analizados, no se evidenciaron diferencias significativas entre las muestras analizadas. Para el caso de las bebidas elaboradas a base de vino tinto y vino blanco, no se reportaron diferencias en ninguno de los atributos sensoriales. Los resultados microbiológicos y turbidez se mantuvieron dentro de los rangos establecidos en todas las muestras. Por lo antes expuesto, se puede concluir que, al mantener las características organolépticas en el producto, la innovación realizada resulta satisfactoria al mejorar su eficiencia y mantener el grado de aceptabilidad de sus potenciales consumidores.

Palabras clave: productos vinícolas, evaluación sensorial, innovación, características organolépticas, estabilización microbiológica.

Sumario: 1 Introducción, 2 Materiales y Métodos, 3 Resultados y Discusión, 4 Conclusiones.

Como citar: Peña, M., Peña, S., \& García, J. (2021). Análisis Sensorial como una Herramienta Clave para Innovar en la Industria Vinícola. Revista Tecnológica - Espol, 33(1), 92-103.

http://www.rte.espol.edu.ec/index.php/tecnologica/article/view/800 


\begin{abstract}
Innovation within the food industry is key to achieving the survival of companies, however, new products strictly need some validation processes which aim to reduce the risk of failure when they are launched on the market. Therefore, sensory analysis is an important tool, making possible the measurement of the degree of acceptability in consumers. The main objective of this research was to evaluate the sensory characteristics of three types of wine products through the microfiltration process compared to their pasteurized counterparts. For this development, the sensory judges of the wine company were validated, and subsequently discriminative and descriptive sensory tests were applied to the study samples. In addition, microbiological and turbidity controls were carried out. From the results it can be observed that there were differences in the attributes: olfactory intensity and gustatory intensity in red wine; however, in the analysis of the other sensory parameters, there were no significant differences among the samples. In the case of drinks based on red wine and drinks based on white wine, no differences were reported in any of the sensory attributes. The microbiological results and turbidity of all the samples were kept within the established ranges. Therefore, it can be concluded that, by maintaining the organoleptic characteristics in the product, the innovation carried out within the production process resulted satisfactory by improving its efficiency and maintaining the degree of acceptability of its potential consumers.
\end{abstract}

Keywords: wine products, sensory evaluation, innovation, organoleptic characteristics, microbiological stabilization.

\title{
1. Introducción
}

En un contexto en el cual la salud y la alimentación son una de las principales preocupaciones del ser humano (Alvarez \& Alvarez de Luis, 2009), la innovación alimentaria toma un especial interés entre la población, y, por tanto, el lanzamiento de nuevos productos innovadores es determinante para el crecimiento y la supervivencia de las organizaciones. Sin embargo, su tasa de fracaso es muy alta, parece ser que existen dentro de las empresas dificultades asociadas a un análisis inadecuado del mercado y la incapacidad de comprender las verdaderas necesidades y gustos del consumidor. El fracaso puede ser parte del aprendizaje necesario en el desarrollo de nuevos productos (Cruz \& Puente Castro, 2012), sin embargo, surgen interrogantes con relación a cómo podrían ser reducidas estas tasas de fracaso. En este sentido, la literatura apunta a que, si los investigadores encargados de los procesos de innovación y desarrollo de nuevos productos siguieran una metodología sistemática y lógica que permita revisar, analizar y evaluar cada una de los factores y variables en el ámbito comercial, técnico y financiero, esta tasa de fracaso podría ser reducida de alguna manera (Schnarch, 2009; Cooper et al., 2009; Edgett, 2007).

Específicamente, en la industria de alimentos, cada vez es más grande la necesidad de desarrollar nuevos productos que garanticen la seguridad alimentaria y las exigencias del consumidor en relación con tres aspectos importantes: microbiológicos, fisicoquímicos y sensoriales.

Con el objetivo de contribuir con información relevante y evidencia empírica para el diseño de nuevos alimentos que cumplan con las exigencias del mercado, el presente trabajo de investigación se enfocará en el análisis de las características sensoriales de dos productos de la industria enológica: un vino tinto y un vino blanco, en el cual se implementó una innovación dentro de su proceso productivo, reemplazando el proceso de pasteurización para estabilización microbiológica por un proceso de microfiltración. El reemplazo del proceso de pasteurización busca eliminar un cuello de botella en la línea de producción y un alto porcentaje de desperdicios, pues el aumento de la temperatura del líquido en la botella incide en un incremento de la presión interior, lo que ocasiona ruptura de botellas y expulsión de corchos.

En este contexto, para garantizar que este cambio en el proceso productivo no incida en diferencias perceptibles para el consumidor, se buscó aplicar herramientas que permitan validar la conservación de las características sensoriales en los productos vinícolas. Por lo tanto, se planteó, a partir del uso de pruebas sensoriales discriminativas y descriptivas, identificar la variabilidad en las características organolépticas de las muestras en estudio y anticipar la percepción que el consumidor final podría tener frente a las transformaciones desarrolladas. 
El objetivo de este trabajo fue identificar las cualidades y los defectos que podría presentar el vino, utilizando como instrumento de medición los sentidos (Lawless \& Heymann, 2010). Esto como una estrategia importante para reducir el riesgo en el momento de innovar dentro de esta industria, al establecer criterios de control de calidad y grado de aceptación del mercado (Espinosa, 2007).

\section{Validación de los panelistas sensoriales}

\section{Materiales y Métodos}

El estudio sensorial se llevó a cabo con diez jueces entrenados de la empresa vinícola, a quienes se realizó una validación con el objetivo de garantizar que sus juicios sean homogéneos y reproducibles en tiempo y espacio (NTE-ISO, 2014). Estas pruebas se realizaron en cinco sesiones semanales de aproximadamente 20 minutos cada una; todas estas evaluaciones se desarrollaron a la misma hora (11:00 am), según lo recomendado por la NTE INEN-ISO 6658:2005 (INEN, 2005).

En cada sesión se presentaron dos muestras de un mismo producto (vino tinto propio de la empresa), provenientes de un mismo lote. Al evaluador se le presentó una muestra sin modificaciones y otra modificada (con adición de ácido tartárico) con el objetivo de que los jueces no intuyan las respuestas. Esta fase fue realizada a ciegas para que el catador no se vea influenciado por la procedencia o marca del producto. Los resultados se procesaron con la prueba de correlación Tau de Kendall, con un nivel de significancia del $95 \%$ en el software RStudio.

\section{Plan de muestreo}

La selección de las muestras de vino y bebidas a base de vino para análisis microbiológico y medición del parámetro turbidez se realizó mediante un muestreo no probabilístico por conveniencia NTE INEN 339: 1994 ( INEN, 1994). En cuanto a las pruebas sensoriales, se utilizó un muestreo crítico.

Se tomaron muestras después de cada etapa de filtración del vino: centrifugación, filtración por tierras, filtración por placas; finalmente, se compararon estas entre los procesos de microfiltración y pasteurización. En todas estas etapas se caracterizaron las muestras desde el punto de vista microbiológico y turbidez con el fin de garantizar que el producto cumpla con los requisitos estipulados por la NTE INEN 372: 2016 (INEN, 2016). Sensorialmente se analizaron las muestras después de la microfiltración y la pasteurización. Todos los análisis se realizaron por triplicado.

\section{Análisis microbiológicos y turbidez}

Se analizaron mohos y levaduras viables por siembra en profundidad, según NTE INEN 152910: 2013 (INEN, 2013); parámetro turbidez, en un turbidímetro Hach 2100P (0 - 1000 NTU), con base en la normativa OIV (2009).

\section{Análisis sensorial}

El análisis sensorial se realizó con base en pruebas discriminativas (pruebas triangulares) y pruebas descriptivas.

1) Pruebas discriminativas: se realizó a través de pruebas triangulares, para lo cual se presentaron a los panelistas tres muestras simultáneamente; dos muestras sometidas al proceso de pasteurización y una muestra por microfiltración. Las muestras fueron codificadas con tres cifras aleatorias. Antes de iniciar la cata, se les solicitó analizar e identificar las muestras que presentan diferencias, detallando el parámetro que permitió establecer la disimilitud. Se proporcionó agua y galletas neutras para limpiar el paladar de los catadores. Los datos obtenidos se compararon con las tablas de interpretación estadística con un nivel de confianza del $95 \%$ (NTE-ISO1, 2014).

2) Pruebas descriptivas: para este análisis se presentaron dos muestras a cada catador: A: después del proceso de microfiltración y B: posterior al proceso de pasteurización. A cada evaluador se le solicitó catar en el siguiente orden: 1) Fase visual; 2) Fase olfativa y 3) Fase gustativa. Se utilizó una escala estructurada de 5 puntos. A los datos obtenidos se aplicó un análisis de varianza para determinar si existen diferencias significativas en algún parámetro entre las muestras analizadas. 


\section{Validación del Panel de Jueces}

\section{Resultados y Discusión}

Para garantizar la confiabilidad en los resultados y evitar posibles errores en el proceso de validación del panel de jueces, en relación con las pruebas desarrolladas en la fase visual, fase olfativa y fase gustativa, se eliminaron dos de los diez (10) catadores por no alcanzar el rendimiento requerido. Los ocho (8) restantes emitieron respuestas totalmente homogéneas.

Específicamente en la fase visual se evaluaron los atributos: color, intensidad y limpidez (Tabla 1). En todos los parámetros evaluados, las respuestas que se obtuvieron fueron muy homogéneas, teniendo un valor p de 1 en casi el $80 \%$ de estas; sin embargo, en todos los casos se aceptó la hipótesis. Aspecto de importancia, pues demuestra que la totalidad de los jueces concuerdan en todos los parámetros evaluados en la fase visual con un nivel de confianza del $95 \%$. Resultado que avala la preparación y el entrenamiento de los jueces para el proceso de análisis sensorial -visual de los vinos.

En la fase olfativa los atributos evaluados fueron intensidad, calidad y aroma, respectivamente (Tabla 2). En los dos primeros parámetros se aprecia homogeneidad en las respuestas, no obstante, en el tercer parámetro (aroma), se obtuvieron dos valores inferiores al p-crítico $(0,05)$, lo que significó rechazar la hipótesis. Estos valores demuestran que dos (2) de los jueces no están lo suficientemente entrenados con respecto a la distinción de aromas.

Tabla 1

Resultados prueba 'Tau de Kendall". Fase Visual

\begin{tabular}{cccccccccc}
\hline \multirow{2}{*}{ Jueces } & \multicolumn{3}{c}{ Color } & \multicolumn{3}{c}{ Intensidad Visual } & \multicolumn{2}{c}{ Limpidez } \\
\cline { 2 - 9 } & $\boldsymbol{p}$-value & $\boldsymbol{p}$-crítico & Conclusión & $\boldsymbol{p}$-value & $\boldsymbol{p}$-crítico & Conclusión & $\boldsymbol{p}$-value & $\boldsymbol{p}$-crítico & Conclusión \\
\hline AB & 1 & 0.05 & SE ACEPTA & 1 & 0.05 & SE ACEPTA & 1 & 0.05 & SE ACEPTA \\
AC & 1 & 0.05 & SE ACEPTA & 1 & 0.05 & SE ACEPTA & 1 & 0.05 & SE ACEPTA \\
AD & 1 & 0.05 & SE ACEPTA & 1 & 0.05 & SE ACEPTA & 1 & 0.05 & SE ACEPTA \\
AE & 0.07 & 0.05 & SE ACEPTA & 0.13 & 0.05 & SE ACEPTA & 1 & 0.05 & SE ACEPTA \\
AF & 1 & 0.05 & SE ACEPTA & 0.13 & 0.05 & SE ACEPTA & 0.68 & 0.05 & SE ACEPTA \\
AG & 1 & 0.05 & SE ACEPTA & 1 & 0.05 & SE ACEPTA & 0.41 & 0.05 & SE ACEPTA \\
AH & 1 & 0.05 & SE ACEPTA & 1 & 0.05 & SE ACEPTA & 1 & 0.05 & SE ACEPTA \\
AI & 1 & 0.05 & SE ACEPTA & 1 & 0.05 & SE ACEPTA & 1 & 0.05 & SE ACEPTA \\
AJ & 1 & 0.05 & SE ACEPTA & 1 & 0.05 & SE ACEPTA & 1 & 0.05 & SE ACEPTA \\
\hline
\end{tabular}

Nivel de significancia del $95 \%$

Fuente: Elaboración propia

Tabla 2

Resultados prueba 'Tau de Kendall". Fase Olfativa

\begin{tabular}{cccccccccc}
\hline \multirow{2}{*}{ Jueces } & \multicolumn{3}{c}{ Intensidad Olfativa } & \multicolumn{2}{c}{ Calidad } & \multicolumn{2}{c}{ Aroma } \\
\cline { 2 - 9 } & $\boldsymbol{p}$-value & $\boldsymbol{p}$-crítico & Conclusión & $\boldsymbol{p}$-value & $\boldsymbol{p}$-crítico & Conclusión & $\boldsymbol{p}$-value & $\boldsymbol{p}$-crítico & Conclusión \\
\hline AB & 1 & 0.05 & SE ACEPTA & 1 & 0.05 & SE ACEPTA & 1 & 0.05 & SE ACEPTA \\
AC & 0.68 & 0.05 & SE ACEPTA & 1 & 0.05 & SE ACEPTA & 0.41 & 0.05 & SE ACEPTA \\
AD & 1 & 0.05 & SE ACEPTA & 1 & 0.05 & SE ACEPTA & 0.13 & 0.05 & SE ACEPTA \\
AE & 1 & 0.05 & SE ACEPTA & 1 & 0.05 & SE ACEPTA & 0.03 & 0.05 & SE RECHAZA \\
AF & 1 & 0.05 & SE ACEPTA & 1 & 0.05 & SE ACEPTA & 0.02 & 0.05 & SE RECHAZA \\
AG & 1 & 0.05 & SE ACEPTA & 1 & 0.05 & SE ACEPTA & 0.23 & 0.05 & SE ACEPTA \\
AH & 1 & 0.05 & SE ACEPTA & 1 & 0.05 & SE ACEPTA & 1 & 0.05 & SE ACEPTA \\
AI & 1 & 0.05 & SE ACEPTA & 1 & 0.05 & SE ACEPTA & 1 & 0.05 & SE ACEPTA \\
AJ & 1 & 0.05 & SE ACEPTA & 1 & 0.05 & SE ACEPTA & 1 & 0.05 & SE ACEPTA \\
\hline
\end{tabular}

Nivel de significancia del $95 \%$

Fuente: Elaboración propia

En la fase gustativa, a partir de los resultados alcanzados en la evaluación de los parámetros acidez, intensidad y naturaleza (Tabla 3 ) se evidenció la homogeneidad en todas las respuestas emitidas por los jueces. 
Tabla 3

Resultados prueba 'Tau de Kendall". Fase Gustativa

\begin{tabular}{|c|c|c|c|c|c|c|c|c|c|}
\hline \multirow[b]{2}{*}{ Jueces } & \multicolumn{4}{|c|}{ Intensidad Gustativa } & \multicolumn{3}{|c|}{ Acidez } & \multicolumn{2}{|c|}{ Naturaleza } \\
\hline & $p$-value & $\begin{array}{c}p- \\
\text { crítico }\end{array}$ & Conclusión & $p$-value & p-crítico & Conclusión & $p$-value & $\begin{array}{c}p- \\
\text { crític } \\
o\end{array}$ & Conclusión \\
\hline AB & 1 & 0.05 & SE ACEPTA & 1 & 0.05 & SE ACEPTA & 1 & 0.05 & SE ACEPTA \\
\hline AC & 1 & 0.05 & SE ACEPTA & 1 & 0.05 & SE ACEPTA & 1 & 0.05 & SE ACEPTA \\
\hline AD & 1 & 0.05 & SE ACEPTA & 1 & 0.05 & SE ACEPTA & 1 & 0.05 & SE ACEPTA \\
\hline $\mathbf{A E}$ & 1 & 0.05 & SE ACEPTA & 0.13 & 0.05 & SE ACEPTA & 1 & 0.05 & SE RECHAZA \\
\hline AF & 1 & 0.05 & SE ACEPTA & 1 & 0.05 & SE ACEPTA & 1 & 0.05 & SE RECHAZA \\
\hline AG & 1 & 0.05 & SE ACEPTA & 1 & 0.05 & SE ACEPTA & 1 & 0.05 & SE ACEPTA \\
\hline $\mathbf{A H}$ & 1 & 0.05 & SE ACEPTA & 1 & 0.05 & SE ACEPTA & 1 & 0.05 & SE ACEPTA \\
\hline AI & 1 & 0.05 & SE ACEPTA & 1 & 0.05 & SE ACEPTA & 1 & 0.05 & SE ACEPTA \\
\hline $\mathbf{A J}$ & 1 & 0.05 & SE ACEPTA & 0.13 & 0.05 & SE ACEPTA & 1 & 0.05 & SE ACEPTA \\
\hline
\end{tabular}

Fuente: Elaboración propia

\section{Análisis microbiológicos}

Para el caso de mohos y levaduras, se ejecutaron en cinco batch durante cada una de las etapas del proceso de filtración. Estas se realizaron en cada uno de los productos en estudio.

Se evidenció una reducción de la carga microbiana de mohos y levaduras en los productos estudiados; los resultados se muestran en la Tabla 4. En la misma se exponen las diferentes concentraciones de microorganismos a medida que al producto en cuestión se le somete a varias etapas de filtración, hasta llegar, inclusive, a la pasteurización.

Tabla 4

Recuento microbiológico de mohos y levaduras durante todas las etapas de filtración del vino (valores promedios)

\begin{tabular}{ccccc}
\hline \multirow{2}{*}{ Etapas } & \multirow{2}{*}{ Unid. } & \multicolumn{3}{c}{ Producto } \\
\cline { 3 - 5 } & & $380(47)$ & $670(70)$ & $690(46)$ \\
\hline Después & & 368.24 & \\
Centrifugación & $168.6(40)$ & $(60)$ & $420.4(41)$ \\
Filtración tierras & & $60.8(12)$ & $164.7(32)$ & $268.7(27)$ \\
Filtración placas & UFC. $250 \mathrm{ml}^{-1}$ & $3(1)$ & $31.7(14)$ & $93.9(31)$ \\
Microfiltración & & $0(0)$ & $0(0)$ & $0(0)$ \\
Después & & & &
\end{tabular}

Nota. V1: Vino tinto seco, V2: Bebida a base de vino blanco, V3: Bebida a base de vino tinto, (): Desviación estándar

Fuente: Elaboración propia

Las etapas que tienen la finalidad de eliminar partículas gruesas y no la reducción total de la carga microbiana evidencian una elevada carga microbiológica en el producto en cuestión. Suarez \& Baldomero (2004) afirman en su investigación que "la centrifugación aplicada como procedimiento único no separa totalmente toda la carga microbiana existente en el vino, por tanto, sirve solamente para disminuir el número de microorganismos y retardar unos días el inicio de la fermentación". Valores promedios de 380 UFC. 250 ml-1(1.52 UFC. ml-1) de vino tinto seco (V1), 670 UFC. $250 \mathrm{ml}-1$ (2.68 UFC. ml-1) de bebida a base de vino blanco (V2) y 690 UFC. 250 ml-1(2.76 UFC. ml-1) de bebida a base de vino tinto (V3) fueron determinados después de la centrifugación. Puede apreciarse que mayores concentraciones se obtienen en V2 y V3, por tratarse de bebidas con un alto contenido de azúcar y bajo grado alcohólico. 
Las concentraciones promedio de 168.6 UFC. 250 ml-1 (0.674 UFC. ml-1) de V1 fueron determinadas después de la etapa de filtración a través de tierras diatomeas (Tabla 4). Estos valores, que discrepan de lo expuesto por Suárez \& Baldomero (2004), afirman que la carga microbiana después de la filtración a través de tierras diatomeas en los vinos tintos, generalmente tiene poblaciones viables de aproximadamente 100 UFC. ml-1. Los valores de cargas microbianas bajas, a comparación con otros autores, fueron posibles obtener debido a la adición como conservante del metabisulfito de sodio (Na2S2O5) en concentración de SO2 libre (30 - 40 ppm); no obstante, el vino aún no logra tener una estabilización microbiológica completa.

Las cargas microbiológicas obtenidas para los productos V2 (368.24 UFC. 250 ml-1 (1.47 UFC. ml-1)) y V3 (420.4 UFC. 250 ml-1 ((1.68 UFC. ml-1)), luego de la etapa de filtración a través de tierras diatomeas, se encuentran por encima de los niveles de V1, si comparamos los resultados de los tres productos estudiados. Sin embargo, y aunque no se logró una estabilización microbiológica, las concentraciones alcanzadas para V2 y V3 son sumamente bajas al compararlas con el resultado de otros autores. Esto se evidencia en la investigación reportada por Ribéreau-Gayon et al. (2006), en la que se señala que, al filtrar este tipo de bebidas por tierras diatomeas, se obtiene cargas promedio de 1250 UFC. 250 ml-1 (5.0 UFC. ml-1); niveles que se encuentran estrechamente vinculados a la permeabilidad de las tierras utilizadas en el proceso, puesto que, mientras menor sea la permeabilidad de estas, se podrá alcanzar cargas microbianas más bajas.

Posteriormente a la etapa de filtración por placa se determinaron cargas microbiológicas promedio de 60.8 UFC. 250 ml-1 (0.24 UFC. ml-1), 164.7 UFC. 250 ml-1 (0.66 UFC. ml-1) y 268.7 UFC. 250 ml-1 (1.07 UFC. ml-1) en V1, V2 y V3, respectivamente. Valores notablemente pequeños se obtienen dado que esta etapa se integra al final del tratamiento global de estabilización antes del embotellado, con el fin de acondicionar el vino para la microfiltración (Tabla 4).

Valores de carga microbiana promedio de 3 UFC. 250 ml-1 (0.01 UFC. ml-1) para V1, 31.7 UFC. 250 ml-1 (0.012 UFC. ml-1) para V2 y de aproximadamente 94 UFC. 250 ml-1 (0.38 UFC. ml1) para V3 fueron obtenidos luego del proceso de microfiltración (Tabla 4). Estos niveles de concentraciones (V1, V2 y V3) justifican la estabilización microbiológica alcanzada, concordando con lo planteado por Ribéreau-Gayon et al. (2006), que mencionan que "la microfiltración por membrana justo antes del embotellado se describe convencionalmente como baja en nivel de microorganismo": NTE INEN 2802:2015 (INEN, 2015), la cual establece un máximo de 10 UFC. ml-1.

En la etapa de pasteurización, los tres productos en estudio (V1, V2 y V3) reportaron ausencia total de carga microbiana para todas las muestras, debido a la relación temperatura-tiempo aplicada que oscila entre 55 y $60^{\circ} \mathrm{C}$ durante 25 minutos, aproximadamente, que es la óptima para la destrucción total de mohos y levaduras.

\section{Turbidez}

Determinaciones del parámetro Turbidez en los tres productos estudiados evidencian que luego del proceso de centrifugación los valores alcanzados fueron 11.32, 4.08 y 4.07 NTU para V1, V2 y V3, respectivamente. Estos valores continúan descendiendo hasta alcanzar niveles de 0.9, 0.72 y $1.12 \mathrm{NTU}$ después del proceso de microfiltración (Tabla 5).

Estas concentraciones presentadas por los tres productos V1, V2 y V3, luego de la cuarta etapa o proceso (microfiltración), indican que se partió de un vino (bebida) totalmente turbio y se logró un vino brillante, encontrándose todos ellos en el intervalo de turbidez de $0.4-1.5 \mathrm{NTU}$, tal como lo indica Vilavella (1998) y Lafuente (2013).

Cabe destacar que la turbidez final en la bebida a base de vino blanco (V2) 0.66 NTU es menor que la bebida a base de vino tinto (V3) 0.89 NTU, debido a que el vino blanco por naturaleza tiene menor cantidad de partículas sólidas (Tabla 5). 
Tabla 5

Medición del parámetro turbidez en tres productos durante todas las etapas de filtración del vino

\begin{tabular}{cccc}
\hline \multirow{2}{*}{ ETAPAS } & V1 & V2 & V3 \\
\cline { 2 - 4 } & Promedio & Promedio & Promedio \\
\hline Después Centrifugación & $11.3(0.4)$ & $4.08(0.3)$ & $4.07(0.3)$ \\
Filtración Tierras & $2.63(0.24)$ & $2.07(0.19)$ & $2.50(0.28)$ \\
Filtración Placas & $1.57(0.07)$ & $1.17(0.12)$ & $1.81(0.09)$ \\
Microfiltración & $0.90(0.06)$ & $0.72(0.01)$ & $1.12(0.07)$ \\
Después Pasteurización & $0.78(0.02)$ & $0.66(0.01)$ & $0.89(0.03)$
\end{tabular}

Nota. V1: Vino tinto seco, V2: Bebida a base de vino blanco, V3: Bebida a base de vino tinto, (): Desviación estándar Fuente: Elaboración propia

Con estos niveles de turbidez alcanzados, tanto para vinos tintos como para bebidas con un contenido de azúcar residual, se puede concluir que, por medio del proceso de microfiltración, se obtienen vinos totalmente brillantes, lo que convierte a este parámetro en sinónimo de calidad, corroborando lo que puede conseguir el proceso de microfiltración en los productos vínicos listos para su comercialización.

\section{Análisis Sensorial}

1) Pruebas Discriminativas: resultados obtenidos en una prueba triangular a través de pruebas discriminativas se presentan en la Tabla 6. Estas evaluaciones se realizaron con el objetivo de analizar la posible influencia de los procesos estudiados (microfiltración y pasteurización) sobre las características organolépticas de los productos.

De los resultados obtenidos, se pudo evidenciar que en el producto V1, 6 de los 8 jueces fueron capaces de distinguir la muestra heterogénea, cumpliendo con lo propuesto en las tablas de interpretación estadística, con un nivel de confianza del $95 \%$ (NTE-ISO1, 2014). Por lo antes expuesto, se puede afirmar que existe una diferencia entre las dos muestras sometidas a los distintos tratamientos. Otro comportamiento se observó en los resultados expuestos para los productos V2 y V3, en los cuales se obtuvieron números de respuestas correctas inferiores a 6 , por lo que no puede afirmase la existencia de diferencias entre las muestras analizadas (Tabla 6).

Tabla 6

Prueba triangular en vino tinto seco, bebida a base de vino blanco y bebida a base de vino tinto

\begin{tabular}{ccccc}
\hline Muestra & $\begin{array}{c}\text { Número de } \\
\text { jueces }\end{array}$ & $\begin{array}{c}\text { Número mínimo } \\
\text { para establecer } \\
\text { diferencias }\end{array}$ & Resultados & Diferencia \\
\hline V1 & & 6 & Sí \\
V2 & 8 & 6 & 2 & No \\
V3 & & & 3 & No \\
\hline
\end{tabular}

Nota. V1: Vino tinto seco, V2: Bebida a base de vino blanco, V3: Bebida a base de vino tinto. Nivel de confianza de los análisis $95 \%$ Fuente: Elaboración propia

2) Pruebas Descriptivas: características organolépticas de tres productos estudiados y sometidos a los dos procesos de estabilización microbiológica (microfiltración y pasteurización) fueron analizadas con el afán de evidenciar si existen diferencias significativas entre las muestras, y, de este modo, constatar si hubo o no afectación en sus atributos sensoriales. Las comparaciones de las características sensoriales fueron: Color, Intensidad Visual, Limpidez, Intensidad Olfativa, Calidad, Aroma, Intensidad Gustativa, Acidez, Naturaleza y Armonía General para cada uno de los tres 
productos en estudio (a: Vino Tinto - V1, b: Bebida a Base de Vino Blanco - V2, c: Bebida a Base de Vino Tinto - V3) se presentan en la Figura 1a), b) y c).

Los resultados que se muestran para V1 (Figura 1a) evidencian diferencias marcadas entre ambos procesos. Partiendo de que se considera que para un vino tinto es importante mantener la tendencia a un color rojo púrpura (color esperado), analizando el color como primer parámetro considerado en este análisis; se observa una ligera diferencia entre los dos procesos, teniendo el vino tinto pasteurizado una menor puntuación, al tener una menor tendencia al color rojo púrpura.

Un aspecto que se tiene que destacar en este fenómeno lo constituyen las altas temperaturas que son aplicadas en la pasteurización. Como resultado del calor aplicado, ocurren procesos de transformación de los compuestos fenólicos (principalmente, antocianinas, responsables del color rojo), que tornan el color a tonalidades amarillentas, quitándole la vivacidad de un vino tinto joven. Comportamiento que concuerda con lo expresado en la investigación realizada por Peña (2006), quien señala que "las temperaturas elevadas favorecen a la pérdida del color en vinos tintos".

El análisis de los parámetros intensidad visual y limpidez (Figura 1a) arroja que no se observan diferencias significativas; esto debido a que ambos parámetros dependen de la cantidad de materia colorante y de los turbios presentes en el producto. Por lo tanto, someter al producto a procesos de eliminación de partículas sólidas con suficiente eficacia provoca que no se vean afectadas las características en cuestión. Comportamiento similar lo demuestran los atributos calidad y naturaleza; ambos se encuentran dentro de los estándares establecidos para estos parámetros.

Valores que coinciden con los hallazgos encontrados por Vilavella (1998), quien menciona que después del proceso de microfiltración organolépticamente no hay diferencias significativas en la calidad con respecto a vinos tratados con la tecnología tradicional. En relación con el parámetro acidez, se observa que no hay diferencia entre las muestras, pues por ambos procesos se obtienen vinos con acidez moderada (Figura 1a).

El parámetro intensidad olfativa demuestra que existen diferencias, en las que el vino tinto pasteurizado ostenta una mayor valoración que el microfiltrado (Figura 1a).

Lo mismo ocurre en relación con el parámetro aroma; el vino, al ser pasteurizado, tiene mayor puntuación que el vino microfiltrado, por lo que el aroma es mucho más intenso (Figura 1a). Ese comportamiento se debe a las altas temperaturas aplicadas, pues vuelven más volátiles a los aromas, aumentando así su intensidad olfativa, así como al efecto que tiene la temperatura aplicada en el proceso de pasteurización, por lo que productos tratados por microfiltración son menos intensos y aromáticos. Similar comportamiento fue señalado por Vilavella (1998), quien expresa que la microfiltración acelera los fenómenos de condensación taninos-antocianos, disminuyendo así la cantidad de coloides que son soporte de los aromas; esto se relaciona a lo citado por Matheus (2004), que menciona que la filtración ocasiona la pérdida de aroma debido a que las moléculas de este se unen a macromoléculas, como las proteínas de la cáscara o de las pepas de la uva, que luego son eliminadas con la turbidez. En cuanto a la intensidad gustativa, también es superior después del proceso de pasteurización, puesto que la temperatura exalta todas estas sensaciones, volviendo a los vinos más ardientes y alcohólicos (Figura 1a).

El análisis comparativo de las características sensoriales de los productos V2 y V3, sometidos al proceso de pasteurización y al proceso de microfiltración, se muestra en la Figura 1b) y Figura 1c). Es importante esclarecer que ambos productos contienen un contenido residual de azúcar del $10 \%$. Específicamente, los resultados obtenidos para V2 muestran que existen mínimas diferencias entre los dos procesos. Como se observa en la Figura 1b), en la mayoría de los parámetros estudiados (Color, Intensidad Visual, Limpidez, Calidad y Aroma) no se observan diferencias significativas, por lo que dichos parámetros no se ven afectados por la pasteurización ni la microfiltración; no obstante, la acidez registra una leve diferencia al ser esta mayor en el proceso de pasteurización. Es conocido que con un aumento en la temperatura se potencializan los sabores ácidos. 
La Figura 1c) muestra las ligeras diferencias entre los procesos estudiados (pasteurización y microfiltración) comparando las características sensoriales del producto V3. En este caso se observan diferencias en la mayoría de los parámetros, teniendo una alta relación con los resultados encontrados en el vino tinto seco (Figura 1a), ya que precisamente V3 es una bebida producida a base de un vino tino, por tanto, el análisis será similar; no obstante, el grado de diferencia que existe entre los dos productos analizados en este caso es inferior.

Finalmente, una vez analizados los diferentes atributos, en el momento de determinar la armonía general y naturaleza de estos, el panel de jueces puntuó con una calificación más alta al proceso de microfiltración para los tres productos V1, V2 y V3.

\section{Figura 1}

Comparación de las características sensoriales entre un proceso de pasteurización y microfiltración de los productos estudiados en una escala hedónica de cinco puntos: a) vino tinto - v1, b) bebida a base de vino blanco - v2, c) bebida a base de vino tinto $-v 3$

a)

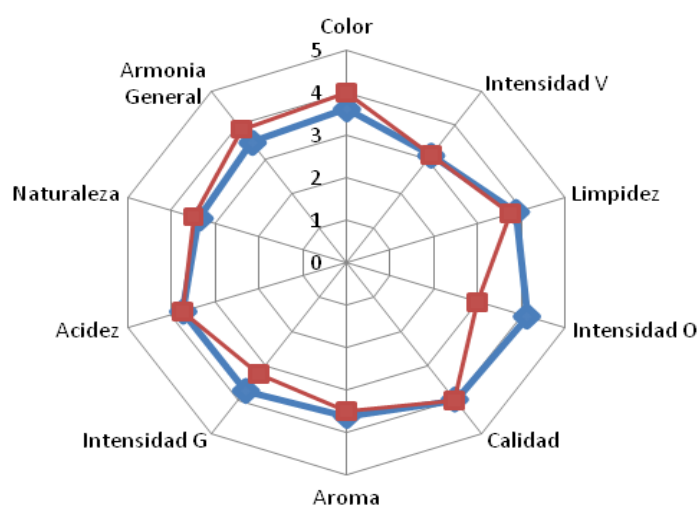

b)

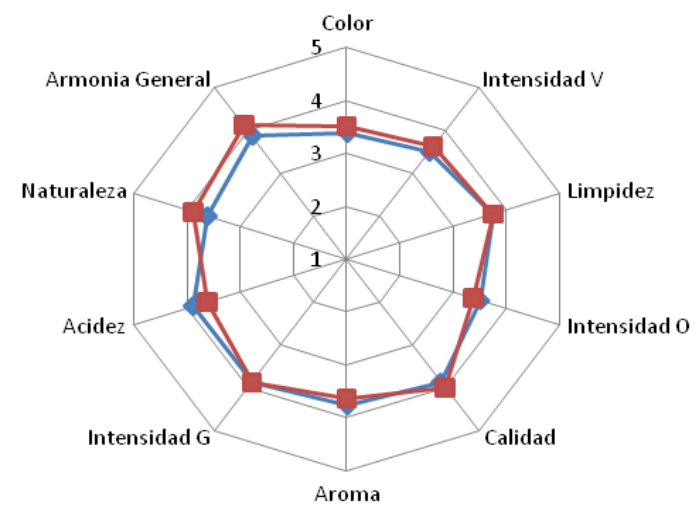

c)

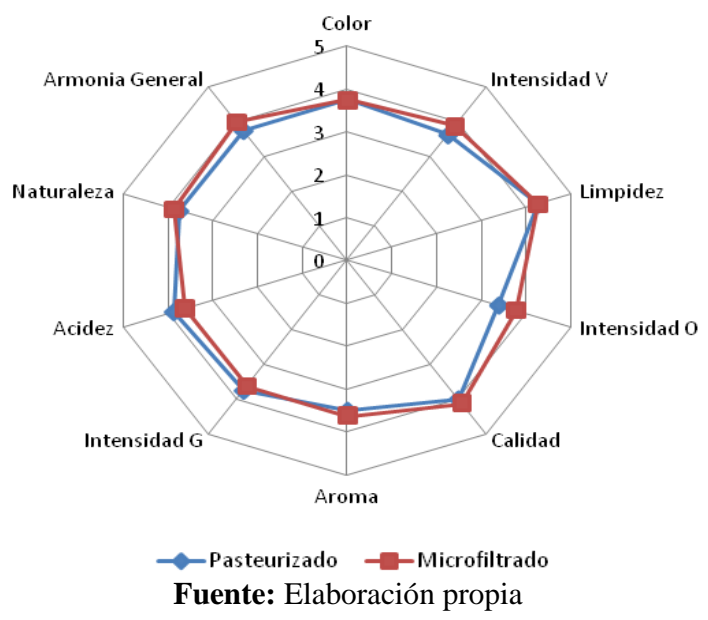

Pruebas sensoriales analizadas en el software estadístico IBM SPSS, mediante un análisis de varianza ANOVA aplicada a los productos, se muestran en la Tabla 7.

El análisis de los resultados expuestos en la Tabla 7 muestra que para el caso del producto V1, de las once (11) características que se sometieron a exámenes, solo en dos (2) de ellas, intensidad olfativa e intensidad gustativa, existen diferencias significativas luego del proceso de microfiltración y pasteurización. Estos hallazgos pueden ser atribuidos al hecho de que el proceso de pasteurización involucra altas temperaturas, lo que podría incidir en la volatilización de ciertas sustancias químicas 
que le atribuyen en el perfil aroma y sabor. Para los demás atributos no se encontraron diferencias significativas después de los procesos.

Tabla 7

Análisis de varianza ANOVA realizado a los productos

\begin{tabular}{|c|c|c|c|c|c|c|c|c|c|}
\hline \multirow{2}{*}{ Característica } & \multicolumn{3}{|c|}{ Vino Tinto (V1) } & \multicolumn{3}{|c|}{$\begin{array}{c}\text { Bebida a Base de Vino Blanco } \\
(\text { V2) }\end{array}$} & \multicolumn{3}{|c|}{$\begin{array}{c}\text { Bebida a Base de Vino Tinto } \\
(\text { V3) }\end{array}$} \\
\hline & $\begin{array}{c}\text { Estadístico } \\
\text { F }\end{array}$ & Sig. & Conclusión & $\begin{array}{c}\text { Estadístico } \\
\text { F }\end{array}$ & Sig. & Conclusión & $\begin{array}{c}\text { Estadístico } \\
\text { F }\end{array}$ & Sig. & Conclusión \\
\hline Color & 0.23 & 0.64 & NO & 0.00 & 1.00 & NO & 0.15 & 0.71 & NO \\
\hline $\begin{array}{l}\text { Intensidad } \\
\text { Visual }\end{array}$ & 0.00 & 1.00 & NO & 0.52 & 0.48 & NO & 0.15 & 0.71 & NO \\
\hline Limpidez & 0.07 & 0.79 & NO & 0.00 & 1.00 & NO & 0.00 & 1.00 & NO \\
\hline $\begin{array}{l}\text { Intensidad } \\
\text { Olfativa }\end{array}$ & 24.65 & 0.00 & SI & 2.33 & 0.15 & NO & 0.00 & 1.00 & NO \\
\hline Calidad & 0.00 & 1.00 & NO & 1.24 & 0.29 & NO & 2.33 & 0.15 & NO \\
\hline Aroma & 0.93 & 0.35 & NO & 1.07 & 0.32 & NO & 0.26 & 0.62 & NO \\
\hline $\begin{array}{l}\text { Intensidad } \\
\text { Gustativa }\end{array}$ & 4.67 & 0.05 & SI & 0.26 & 0.62 & NO & 1.00 & 0.33 & NO \\
\hline Acidez & 0.00 & 1.00 & NO & 2.33 & 0.15 & NO & 11.67 & 0.06 & NO \\
\hline Naturaleza & 0.23 & 0.64 & NO & 0.37 & 0.55 & NO & 2.74 & 0.12 & NO \\
\hline $\begin{array}{l}\text { Armonía } \\
\text { General }\end{array}$ & 1.61 & 0.22 & $\mathrm{NO}$ & 0.64 & 0.44 & NO & 3.50 & 0.08 & NO \\
\hline
\end{tabular}

Fuente: Elaboración propia

Para los productos V2 y V3, los resultados de la prueba (Tabla 7) muestran que no existen diferencias significativas para ninguno de los atributos estudiados.

Podría ser que, por el hecho de utilizar altas temperaturas, se volatilizan ciertos componentes $y$, en cuanto al sabor, se pierden ciertas sustancias que dan su cualidad a este.

\section{Conclusiones}

Este trabajo busca contribuir en los estudios que se realizan para la sustitución de etapas o procesos (en planta) durante la producción vinícola, así como en potencializar el panel sensorial como herramienta para validar procesos innovadores en la industria.

La capacitación continua a los jueces que forman parte del panel sensorial es imprescindible; para ello, se decidió establecer un plan de capacitación de identificación de aromas; este plan posibilita que las autoridades en cuestión puedan distinguir de una mejor manera los aromas característicos en los vinos que se evalúan.

Se constató que el proceso de microfiltración es capaz de sustituir al proceso de pasteurización, otorgando un aspecto totalmente brillante y una estabilización microbiológica adecuada, tanto en vinos tintos y secos, así como en bebidas a base de vino con un contenido de azúcar residual del $10 \%$.

El uso del panel sensorial posibilita determinar que el proceso de microfiltración no afecta las características organolépticas en bebidas a base de vino tinto y vino blanco con un contenido de azúcar residual del $10 \%$. 
El análisis sensorial se puede utilizar como una herramienta que permita validar productos vinícolas o procesos innovadores antes de ser lanzados en el mercado. Para este estudio, se pudo verificar que la innovación realizada en el proceso productivo (microfiltración) es viable, pues resulta más eficiente y, además, ayuda a conservar las características organolépticas de los productos.

\section{Referencias}

Álvarez Munárriz L, Álvarez de Luis A. (2009). Estilos de vida y alimentación. Gazeta de Antropología. 25(27), http://www.gazeta-antropologia.es/?p=1916

Cooper, Robert G.; Edgett, Scott J. (2009). Generating Breakthrough New Product Ideas: Feeding the Innovation Funnel. Product Development Institute.

Cruz, M. L., y Puente Castro, R. (2012). ¿Hay verdadera innovación en los lanzamientos de nuevos productos? Factores relevantes de éxito y fracaso en el caso colombiano. Estudios Gerenciales, 28(EE), 263-280.

Edgett, S. (2007). Portfolio Management: Optimizing for Success. Houston: American Productivity \& Quality Center.

Espinosa, M. J. (2007) Evaluación Sensorial de los Alimentos. Versión digital. Editorial Universitaria, Cuba. https://s47003acac0f1f7a3.jimcontent.com/download/version/1463707242/module/8586131883/name/ LIBRO\%20ANALISIS\%20SENSORIAL-1\%20MANFUGAS.pdf

INEN (1994). Norma Técnica Ecuatoriana NTE INEN 339. Bebidas alcohólicas. Muestreo.

INEN (2005). Norma Técnica Ecuatoriana ISO 6658. Análisis sensorial de alimentos. Metodología. Guía general.

INEN (2013). Norma Técnica Ecuatoriana NTE INEN 1529-10. Control microbiológico de los alimentos. Mohos y levaduras viables. Recuentos en placa por siembra en profundidad.

INEN (2015). Norma Técnica Ecuatoriana NTE INEN 2802. Bebidas Alcohólicas, Cocteles o bebidas alcohólicas mixtas y los aperitivos. Requisitos.

INEN (2016). Norma Técnica Ecuatoriana NTE INEN 372. Bebidas alcohólicas. Vino. Requisitos.

Lafuente, S. N. (2013). Efectos de la microfiltración tangencial en el color del vino de mora de castilla. BSc. [Tesis de grado, Universidad Tecnológica Equinoccial].

http://repositorio.ute.edu.ec/handle/123456789/5000

Lawless, H. T., \& Heymann, H. (2010). Sensory evaluation of food: principles and practices. Springer Science \& Business Media.

Matheus, M. C. (2004). Estudio de factibilidad para la producción de Uvas (Vitis vinífera), vino y vinagre en Imbabura. BSc. [Tesis de grado, Universidad San Francisco de Quito)

NTE-ISO1 (2014). Norma Técnica Ecuatoriana ISO 4120. Análisis sensorial. Metodología. Prueba Triangular

NTE-ISO (2014). Norma Técnica Ecuatoriana ISO 8586-2. Análisis sensorial. Guía general para la selección, entrenamiento y control de evaluadores. Parte 2: Evaluadores sensoriales expertos.

OIV (2009). Compendium of International Methods of Analysis of Wines and Musts OIV-MA-AS2-08. Wine turbidity.

Peña, A. (2006). El Color de los vinos. Informe Técnico. Vendimia, 24 - 26. Corporacion Chilena del vino.

Ribéreau-Gayon, P., Glories, Y., Maujean, A. and Dubourdieu, D. (2006). Clarifying Wine by Filtration and Centrifugation. In Handbook of Enology (eds P. Ribéreau-Gayon, Y. Glories, A. Maujean and D.

Dubourdieu). https://doi.org/10.1002/0470010398.ch11 
Schnarch K., Alejandro. (2009). Desarrollo de Nuevos Productos y Empresas: Creatividad, Innovación Y Marketing, como generar, desarrollar e introducir con éxito nuevos productos, servicios o empresas. MC GRAW-HILL. 409 P. / 24 CM. Edición 5.

Suarez, J. A., Baldomero, I. L. (2004). Microbiología enológica: fundamentos de vinificación. Ediciones Mundi-Prensa.

Vilavella Araujo, M. (1998). La Filtración tangencial en el tratamiento de vinos. Universitat de Lleida. 